\title{
Hystérie compliquée d'extase : temps et biens de consommation dans la maladie d'une paysanne savoyarde dans les années 1820
}

"Hysteria complicated by ecstasy" : time and commodities in the malady of a Savoyard peasant girl in the 1820s

Jan Goldstein

\section{CpenEdition}

\section{Journals}

Édition électronique

URL : http://journals.openedition.org/rh19/3873

DOI : 10.4000/rh19.3873

ISSN : $1777-5329$

\section{Éditeur}

La Société de 1848

Édition imprimée

Date de publication : 1 juillet 2009

Pagination : 101-113

ISSN : 1265-1354

\section{Référence électronique}

Jan Goldstein, «Hystérie compliquée d'extase : temps et biens de consommation dans la maladie d'une paysanne savoyarde dans les années 1820 », Revue d'histoire du XIXe siècle [En ligne], 38 | 2009, mis en ligne le 05 septembre 2009, consulté le 03 mai 2019. URL : http://journals.openedition.org/ rh19/3873 ; DOI : 10.4000/rh19.3873 


\section{JAN GOLDSTEIN}

\section{Hystérie compliquée d'extase : temps et biens de consommation dans la maladie d'une paysanne savoyarde dans les années $1820^{1}$}

Ce bref article est le fruit d'un projet de micro-histoire qui m'occupe par intermittence depuis plus de dix ans. Ce projet fut en quelque sorte mon passe-temps intellectuel lorsque je rédigeais The Post-Revolutionary Self ${ }^{2}$ qui m'a longtemps accaparée; c'est finalement devenu un livre, actuellement sous presse $^{3}$. Sa genèse date d'un moment fortuit que j'ai passé dans une bibliothèque parisienne où, en feuilletant distraitement le catalogue imprimé, je suis tombée sur le titre d'un manuscrit daté des années 1820 : «Observations de Nanette Leroux : Hystérie compliquée d'extase $»^{4}$. Attirée par cette étiquette nosologique bizarre et évocatrice, j'ai demandé à voir le manuscrit; il est arrivé, rempli de ratures et d'insertions, difficile à déchiffrer, et, à la fois fascinée et prudente, $j$ 'ai commencé à le lire.

Le manuscrit en question était un texte inédit, une étude de cas sur une jeune paysanne savoyarde diagnostiquée et traitée entre 1822 et 1825 . Comprenant plus d'une centaine de pages, il est beaucoup plus long que les textes qui relèvent du genre de l'étude de cas à cette époque. On pense aux travaux de Philippe Pinel, le père fondateur de la psychiatrie française, et aux «historiettes» squelettiques - c'est le terme qu'il emploie - d'un ou deux paragraphes que l'on trouve sur ses malades dans son Traité médico-philosophique de l'aliénation mentale $(1801)^{5}$. Atypique, le récit de Nanette Leroux l'est

1. J'ai présenté ce texte dans le séminaire de Dominique Kalifa à la Sorbonne en février 2008. Je voudrais remercier Sylvie Garnier, qui l'a généreusement lu avant cette présentation pour en corriger la grammaire et l'usage français.

2. Jan Goldstein, The Post-Revolutionary Self: Politics and Psyche in France, 1750-1850, Cambridge, Massachusetts, et Londres, Harvard University Press, 2005.

3. Jan Goldstein, Hysteria Complicated By Ecstasy: The Case of Nanette Leroux, Princeton et Oxford, Princeton University Press, à paraitre en automne 2009. Ce livre comprend à la fois une longue introduction analytique de quatre chapitres et ma traduction anglaise du texte de l'étude de cas.

4. Bibliothèque de l'Institut de France, manuscrit 2046. J'ai trouvé des parties supplémentaires du texte de l'étude de cas dans le manuscrit 2030. Les origines de ces deux manuscrits séparés et leurs rapports textuels compliqués sont discutés dans Hysteria Complicated By Ecstasy, chapitre 4. Les papiers d'Alexandre Bertrand ont été confiés à la bibliothèque de l'Institut par son fils Joseph, mathématicien qui en est devenu secrétaire perpétuel.

5. Philippe Pinel, Traité médico-philosophique sur l'aliénation, ou la manie, Paris, Richard, Caille et Rivier, An IX/1801, p. 228 et 231-232. 
également car il a deux auteurs : le docteur Charles-Humbert-Antoine Despine (1777-1852), un notable de province, médecin-directeur des thermes, contrôlés par l'État, à Aix-les-Bains et le docteur Alexandre Bertrand (17951831), médecin à Paris, ancien élève de l'École polytechnique et diplômé de la Faculté de médecine de Paris, plus jeune que Despine, assez impécunieux mais «branché» sur le réseau intellectuel de la capitale, écrivain scientifique pour le journal Le Globe, frayant avec le futur premier ministre François Guizot et le vieux philosophe solitaire Maine de Biran. La division du travail entre ces deux praticiens était nette : Despine a traité Nanette Leroux luimême et a pris des notes copieuses sur son comportement et sur les progrès de sa maladie; Bertrand, qui n'a jamais rencontré Nanette, s'est chargé d'une rédaction cohérente de l'étude de cas, utilisant les gros cahiers de son collègue comme matière brute et source principale. Autrement dit, Bertrand était le porte-plume de Despine, lequel avait délibérément cherché un savant de la métropole, présumé plus délié que lui, pour conceptualiser le cas et transformer les notes fragmentées en un récit suivi ${ }^{6}$.

Presque dès le début, le manuscrit m’a passionnée sous plusieurs angles : il donne des renseignements, habituellement si rares, sur l'expérience intime d'une femme des couches inférieures de la société rurale sous la Restauration. Il esquisse à la fois la pratique du magnétisme animal (le précurseur de l'hypnose) et les rapports entre la science parisienne et la science provinciale de cette époque. Il révèle les mœurs villageoises et les attitudes médicales à l'égard de l'acte très ambigu que l'on appelait un attentat à la pudeur. Il pose enfin une question foucaldienne: Nanette Leroux a-t-elle vécu à une époque antérieure à la cristallisation de l'objet discursif de "la sexualité» et, si oui, son étude de cas pourrait-elle nous aider à imaginer concrètement ce que cela voulait dire de vivre dans de telles circonstances?

Après avoir longtemps ruminé le manuscrit, j'ai été également frappée par un autre trait plus global : à savoir la liminalité et du texte et des objets auxquels il fait allusion, leur position intermédiaire, leur indétermination. Chose évidente, le texte lui-même est polyphonique : on entend les voix des deux médecins-auteurs qui sont, de plus, souvent en désaccord, Bertrand se moquant ouvertement de la théorie traditionnelle du magnétisme animal, celle de Mesmer, qu'embrasse son soi-disant collaborateur, et soutenant une théorie carrément révisionniste du même phénomène. Ces deux voix opposées ne sont pas les seules à contribuer à cette polyphonie bruyante. On entend aussi la voix d'un homme improbable, le paysan lettré Mailland qui

6. Despine décrit sa recherche d'un collaborateur dans l'introduction du seul livre qu'il ait jamais publié, De l'emploi du magnétisme animal et des eaux minérales dans le traitement des maladies nerveuses, Paris, Germer Baillière, 1840, un ouvrage qui ne parut que quand Despine avait la soixantaine. Ayant choisi Bertrand pour ce rôle, il lui communiqua «la totalité de (ses) notes" sur les malades nerveuses (y compris sur Nanette Leroux) qu'il avait traitées pendant sa longue carrière médicale. Mais les espoirs des deux médecins furent anéantis par «la mort prématurée» de Bertrand en 1831. Ce malheureux événement "a laissé ce travail inachevé et probablement perdu pour la science» (p. xliii-xliv). 
habite la maison où Nanette est employée comme domestique et qui travaille pour le même maître. Loin d'être le paysan rude et stéréotypé, ce Mailland possède une sensibilité exquise; il entretient un rapport affectif et spontané avec Nanette, ce qui a incité Despine à le charger d'observer la jeune femme et de prendre, lui aussi, des notes copieuses sur le cours de sa maladie. Et dans ce discours polyphonique on entend aussi quelquefois la voix de Nanette elle-même, qui n'a rien écrit mais dont les mots sont directement rapportés par Despine et Mailland.

Également liminales sont les entités - la ville, la région, l'État - qui forment la toile de fond du récit de Nanette. La Savoie, la région francophone où habitaient Despine et Nanette et où s'est déroulé le cas, était, pendant les années 1820 comme avant la Révolution francaise, administrée par la monarchie piémontaise. De la République jacobine jusqu'à la chute de Napoléon, elle avait été française puis le devint définitivement après 1860. Le cadre de l'étude de cas était donc une zone vraiment liminale sur le plan national. Il en était de même de la ville thermale d'Aix-les-Bains, cadre plus limité où Despine essaie de guérir Nanette au moyen de l'hydrothérapie et du magnétisme. Dans les années 1820, Aix-les-Bains et quelques autres villes thermales sont devenues la Mecque des touristes riches - aristocrates et bourgeois, français et étrangers - à la recherche du beau monde (ou de la bonne société) et de vacances salubres ${ }^{7}$. Ces villes constituaient ainsi des poches incongrues de modernité : petites enclaves de gens à la mode parmi des populations agraires. Un homme en visite à Aix en 1829 remarquait ainsi dans les rues de la ville le voisinage de brillants carrosses et de troupeaux de moutons ${ }^{8}$ - emblème parfait de cette liminalité. Enfin, le moment historique était aussi liminal : le rejet complet de la Révolution après 1815 et la restauration d'une monarchie dynastique dans le style de l'Ancien Régime en Savoie n'avait rien de convaincant ni de stable ${ }^{9}$. Cette liminalité multiple confere une vitalité intéressante à cette étude de cas, - la vitalité de quelque chose en mouvement, en pleine éclosion. Elle a fortement aiguisé ma curiosité scientifique pour ce texte.

Mes premiers travaux sur le manuscrit se concentrèrent sur le problème de la sexualité dans une perspective foucaldienne. Je remarquais ainsi plusieurs détails, dont la scène centrale d'auto-guérison, qui me semblaient remplis d'allusions sexuelles non indiquées comme telles. Les hommes qui possédaient le pouvoir interprétatif - c'est-à-dire Despine et Bertrand -

7. Voir à ce sujet Dominique Jarrassé, Les thermes romantiques. Bains et villégiatures en France de 1800 à 1850, Clermont-Ferrand, Institut d'études du Massif central, 1992; Douglas Mackaman, Leisure Settings : Bourgeois Culture, Medicine, and the Spa in Modern France, Chicago, University of Chicago Press, 1998; et Jérôme Penez, Histoire du thermalisme en France au XIX'e siècle. Eau, médecine et loisirs, Paris, Economica, 2005.

8. Comte François-Marie de Fortis, Amélie, ou Voyage à Aix-les-Bains et aux environs, Turin, Pic, 1829, 2 volumes, volume I, p. 3.

9. Voir par exemple Michael Broers, "Sexual Politics and Political Ideology under the Savoyard Monarchy, 1814-21", English Historical Review 114 (1999), p. 607-35. 
semblaient systématiquement inconscients de ce qui, pour nous, lecteurs du début du XXIe siècle, saute aux yeux. J'avancerai donc la thèse que ces médecins étaient, pour ainsi dire, au seuil de la sexualité, dans le sens spécialisé et étroit de ce terme chez Michel Foucault ${ }^{10}$. C'est-à-dire que dans leurs tentatives d'explication scientifique de cette maladie mystérieuse, la chose génitale n'était pas leur option par défaut, pour reprendre un terme courant en informatique de nos jours. Ce n'est pas l'élément qu'ils supposent omniprésent et omnipotent mais caché et qu'ils considèrent a priori comme la clef des symptômes psychopathologiques. Je ne peux pas présenter ici cet argument dans son ensemble mais simplement signaler cet aspect de mon projet ${ }^{11}$. Je dois indiquer aussi que l'expression "être au seuil", que je viens d'employer pour décrire nos deux médecins-auteurs comme «au seuil de la sexualité", est la signification étymologique de la liminalité. La position de ce cas vis-à-vis de l'objet discursif de la sexualité serait donc encore un exemple de sa liminalité.

Cet article présente la partie de ma recherche qui a trait, d'une part, à l'expérience du temps et, d'autre part, au rôle et à la signification des biens de consommation. Il me faut tout d'abord résumer l'histoire de Nanette telle qu'elle est racontée par nos deux auteurs, Despine et Bertrand. Je soulignerai au passage l'un des épisodes principaux du cas qui m'a servi de preuve dans mon argumentation sur la sexualité, mais l'objectif principal, ici, est de transmettre les informations nécessaires pour comprendre mon analyse du rôle du temps et des biens de consommation.

Nanette Leroux était une simple villageoise "de dix-huit ans", "cheveux rouges, peau lentillée, yeux vifs» qui «avait été réglée pour la première fois à quinze ans et demi ». Je reviendrai plus tard sur l'importance de ce dernier fait. Elle avait reçu une éducation supérieure à sa classe sociale, ayant passé un an chez les sœurs de Saint-Joseph à Aix où elle avait appris à lire et à écrire. Employée comme domestique dans son petit village natal situé à six kilomètres d'Aix, ses devoirs comprenaient les tâches ménagères, la couture, la surveillance des chèvres que l'on faisait paitre près de la maison. Elle avait en général joui d'une bonne santé jusqu'à l'été de 1822 quand elle a commencé à présenter toute une gamme de symptômes nerveux : convulsions, défaillances, léthargie, et surtout l'immobilité totale que certains médecins de l'époque, y compris Despine, qualifiaient de catalepsie hystérique. La cause de cette maladie semblait à Nanette transparente et ses médecins avaient ten-

10. Michel Foucault, Histoire de la sexualité, volume 1, La volonté de savoir, Paris, Gallimard, 1976, troisième partie, «Scientia sexualis», en particulier p. 87-94.

11. L'argumentation détaillée sur ce point controversé se trouve dans mon Hysteria Complicated By Ecstasy, en particulier la section du chapitre 2 intitulée "Diagnostics: Catalepsy and Hysteria circa 1820," et celle du chapitre 3, "A Twenty-First-Century Interpretation of the Case: The Place of Sexuality". J'y évoque le concept d'hystérie dans l'acception qu'il recevait au XVIII siècle, son rapport au genre et sa persistance dans l'outillage mental de Despine; je mentionne également le développement au XIX ${ }^{\mathrm{e}}$ siècle d'un discours de "l'instinct" appliqué à l'étiologie de la psychopathologie. 
dance à lui donner raison. Selon Bertrand, «la malade attribuait l'invasion de sa maladie [...] à des frayeurs répétées que lui avait causé un mauvais sujet, un garde champêtre, qui à plusieurs reprises avait voulu attenter à sa pudeur.» On n'avait fait venir le personnel médical qu'en novembre, moment où le docteur Vidal, collègue subalterne du docteur Despine aux thermes d'Aixles-Bains, lui avait rendu visite et avait ordonné «une potion calmante». À cette époque Nanette, se croyant en grand danger, s'était fait confesser et avait demandé à recevoir l'extrême-onction. Mais la mort attendue n'était pas arrivée, au contraire, la maladie de Nanette avait persisté et, tel ce Protée qu'est toujours l'hystérie, elle avait continué de produire des symptômes nouveaux : notamment, à ce stade, la perte momentanée de la capacité de parler et des épisodes de soi-disant "transport des sens", à savoir une migration de l'aptitude sensorielle depuis les organes où elle se situe habituellement vers d'autres parties du corps.

La première percée thérapeutique, qui eut lieu en janvier 1824, était due aux interventions non du docteur Vidal mais du paysan Mailland. «Ce Mailland", Bertrand nous en informe avec une excitation anticipatoire, «va jouer un grand rôle dans l'histoire de notre [malade]». Selon Bertrand, Mailland avait tellement réussi à "rassurer Nanette par la [seule] force de son éloquence villageoise» que la parole lui était aussitôt revenue. Mais cette guérison s'est avérée éphémère. Au début du mois de mars 1824, le docteur Despine visita la malade qu'il trouva atteinte de mutisme et au beau milieu $\mathrm{du}$ "transport des sens». Aussi lui parla-t-il «à la nuque [et] elle répondit par signes ». Despine conseilla alors de l'amener immédiatement à son établissement d'Aix-les-Bains pour suivre une séquence de ses thérapies favorites : bains, douches, électricité, magnétisme. Une semaine plus tard, après son premier bain chaud à Aix, Nanette présenta le «transport des sens» sous une forme extravagante et fleurie. «Les oreilles perdirent la faculté d'entendre et le sens de l'ouïe fut transporté successivement aux coudes, aux seins, à l'épigastre, au bout des doigts. » Nanette éprouvera fréquemment ce transport des sens et ces déplacements anatomiques bizarres suscitent des locutions poétiques chez le rédacteur de l'étude de cas, par exemple : «On tenta inutilement de se faire entendre de la malade en la touchant.»

Pendant la majeure partie de 1824, la vie de Nanette se déroula d'une façon distincte tant sur le plan temporel que géographique. Les périodes de convalescence dans son village, où Mailland la surveillait tendrement, alternèrent avec les périodes de traitements agressifs entre les mains de Despine à Aix. Autre phénomène important : elle commença à émettre des prescriptions pour sa propre guérison et quand son entourage lui fournissait ce qu'elle avait demandé ou avait suivi les procédures qu'elle avait spécifiées en détail, sa condition s'améliorait momentanément. Sa première prescription précisa qu'elle avait besoin d'une montre. Désormais cette montre, qui lui avait été remise par Despine, fera de nombreuses apparitions dans l'étude de 
cas. Selon l'analyse que je présenterai plus tard, cet objet atteste la préoccupation de Nanette pour le temps.

Le tournant pour Nanette eut lieu en septembre 1824. Après avoir annoncé à Despine qu'elle "n'avait plus rien à craindre de sa maladie», elle fut amenée sous l'effet de la cajolerie persistante de son médecin à raconter l'événement «étrange» qui avait modifié sa "manière d'être» et l'avait définitivement soulagée. Deux semaines plus tôt, elle avait pris un bain. L'ayant préparé elle-même et "s'y étant plongée et bien à son aise ", elle avait placé le bâton de cire d'Espagne, un des accessoires ordinaires de la pratique magnétique du docteur Despine que ce dernier avait souvent utilisé dans le traitement de Nanette, «en travers de son bas-ventre de manière que le centre répondait au pubis et les deux bouts aux cuisses, qu'alors ayant à la main un gobelet elle l'avait successivement promené sur les diverses parties du corps. » Lorsqu'elle «l'avait placé comme une ventouse sur le sein gauche, le mamelon au centre du sein, elle avait éprouvé au moment du contact du verre sur le sein une espèce de frémissement universel avec un feu électrique qui lui avait passé dans tout l'intérieur et qu'aussitôt elle s'était trouvée aussi bien qu'avant d'être malade. » À dater de ce jour, elle fut «gaie, active, laborieuse.» Il faut noter qu'en acceptant la réalité de cette auto-guérison, Despine, se demandant comment elle avait réussi, n’a rien soupçonné de sexuel. Il n’a pas interprété le «frémissement universel » comme un orgasme. En fait, il n’a rien interprété du tout. Adepte de l'hydrothérapie, il a plutôt cherché à élucider le bon résultat thérapeutique en demandant à Nanette la température du bain!

Avant Noël de la même année, plusieurs prétendants déclarent vouloir épouser Nanette et quelques-uns consultent le docteur Despine sur son état de santé : le médecin la déclare fondamentalement guérie, en ajoutant que "le mariage ne pouvait lui nuire pourvu qu'on la traitât avec douceur et ménagement». Et Nanette se marie en janvier 1825. Mais le tableau commence à s'assombrir au moment où le journal de Despine - et l'étude de cas - se termine. Nanette, nous dit-on, «ne jouit pourtant pas longtemps de la santé qu'elle n'avait recouvrée qu'avec tant de peine». Enceinte en juillet, dès novembre elle était tout le temps dans un grand désarroi et souvent brouillée avec son mari. De façon menaçante, était reparu le «clou hystérique", c'est-à-dire une douleur passagère comparable à la douleur d'un clou dans la tête, et qui était à cette époque, avec la boule hystérique, la véritable signature de l'hystérie.

Comment comprendre la maladie de Nanette Leroux?

Certes, Despine et Bertrand ont tous les deux leurs propres façons d'aborder cette question, chacun suivant sa théorie médicale préférée. Comme historienne, j'ai, bien sûr, reconstruit avec soin leurs deux modes de compréhension, mais comme lectrice du XXI e siècle je ne les ai trouvés ni l'un ni l'autre très satisfaisants. Nos deux médecins-auteurs se limitent à la question 
assez étroite de déterminer pourquoi certaines interventions thérapeutiques ont réussi à soulager les symptômes de Nanette, au moins momentanément. Pour Despine, la réussite résulte toujours d'une opération physique - le magnétisme ou l'application d'eaux de températures différentes - sur le système de fluide électrique qu'est pour lui l'organisme humain. Pour Bertrand, le succès résulte toujours de forces psychologiques : à savoir des rapports insaisissables d'autorité et de confiance qui lient la malade à ses médecins et ici à son gardien protecteur, Mailland. Ces deux approches si différentes sont néanmoins semblables en ce qu'elles font l'impasse sur les détails riches, abondants et évocateurs du cas. Ainsi même l'attentat à la pudeur qu'a subi Nanette se trouve réduit sous la plume de Despine et Bertrand à une «frayeur» (c'est leur terme, souvent répété) que Nanette a ressentie événement bouleversant, à vrai dire, mais sans contenu spécifique d'aucune sorte. Cette «frayeur» a, selon Despine, ébranlé la distribution de son fluide électrique, nuisant à sa santé; selon Bertrand, elle a rompu sa confiance à l'égard de son entourage - changement psychique qui a entraîné des symptômes physiques. Ni Despine ni Bertrand ne s'interrogent sur les particularités des épisodes mémorables du cas : l'attentat à la pudeur du garde champêtre, la "célèbre scène» dans le bain (c'est leur expression), le souci de Nanette de posséder une montre. Au contraire, mon analyse de la maladie de Nanette essaye de prendre en compte tous ces détails frappants, de les assembler comme les fragments d'un puzzle.

Mon hypothèse est que cette maladie, telle qu'elle est racontée dans l'étude de cas, relève d'une tentative de construction de soi chez cette jeune femme, tentative temporairement efficace mais inévitablement vouée à l'échec. J'interprète le geste du garde champêtre - joué par Nanette dans une scène somnambulique décrite en détail par Mailland dans ses notes - comme une expérience effrayante et sexuelle. Cet attentat, qui a souligné sa nubilité, a nettement posé pour Nanette la question de son destin. Ses propres sentiments érotiques, peut-être même éveillés par l'acte du garde champêtre, ajoutés au rôle traditionnel attendu d'une femme de campagne semblaient lui indiquer que son corps déterminerait son sort. Mais certaines sollicitations engendrées par son milieu, celui du début du XIX siècle, avaient inspiré en elle le souhait rebelle - hésitant et ambivalent à vrai dire - d'une certaine autonomie. Le choc causé par l'attentat à sa pudeur était à la fois une frayeur viscérale - le garde champêtre avait brandi son épée - et la prémonition anxieuse qu'elle n'échapperait jamais à sa condition biologique : celle de n'être qu'un être procréateur. L'efficacité thérapeutique du bain avec le bâton phallique et le frisson orgasmique découlerait donc de la réalisation d'une ambition d'autosuffisance, symbolisée par le plaisir érotique solitaire. Le fait qu'elle ait réalisé son ambition en braconnant sur le terrain de son médecin, qui incarne l'autorité masculine - Despine, hydrothérapeute expert, qui lui donnait des bains et qui manipulait le bâton de cire pour ses expériences 
magnétiques - était, on peut le supposer, une source de satisfaction supplémentaire. En effet, son auto-érotisme s'est doublé de l'auto-guérison, ce qui lui a permis de rompre deux fois sa dépendance à l'égard d'un homme.

Cependant, quels sont les éléments dans l'environnement social de Nanette qui ont incité cette paysanne savoyarde à aspirer à l'autonomie et quelle preuve l'étude de cas fournit-elle d'une telle ambition? On en arrive enfin aux biens de consommation et à l'expérience du temps.

En premier lieu, il est nécessaire de rappeler que la Savoie a vécu les bouleversements de la Révolution française, parmi lesquels des mouvements indigènes en faveur des événements de 1789 en France, l'annexion de la région par la République jacobine, la mise en vigueur rétroactive de l'abolition des seigneuries et de la nationalisation des biens de l'Eglise, les lois républicaines sur la scolarisation des enfants des deux sexes ${ }^{12}$. Née en 1803 , Nanette n’a pas eu l'expérience directe de la période révolutionnaire mais il est bien probable qu'elle en a entendu parler par sa famille. Elle en était une bénéficiaire indirecte : l'école des filles des classes populaires fondée à Aix par les sœurs de Saint Joseph vers la fin de l'époque napoléonienne et où Nanette a appris à lire et à écrire, était une tentative religieuse de poursuivre la scolarisation des filles commencée par l'État durant la première République ${ }^{13}$.

Même si les effets de la Révolution française sur Nanette sont plus ou moins évidents, ceux liés à la "révolution de la consommation" le sont incontestablement plus. Auteur d'une thèse d'État magistrale sur la Savoie au XVIII e siècle, Jean Nicolas ${ }^{14}$ emploie les termes «d'effervescence» et de «dynamisme multiforme» pour décrire le développement socio-économique de la région après 1750 et surtout la croissance d'un marché intérieur et l'expansion du travail salarié. La Savoie était un carrefour européen de colportage; le cabaret, cette nouvelle institution informelle, a surgi de nulle part et a proliféré, jouant, selon Nicolas «un rôle prépondérant dans l'évolution des mœurs". Dans cet espace de sociabilité où l'on servait des boissons alcoolisées et où la clientèle devenait exubérante, les colporteurs vendaient

12. La loi Bouquier du 29 frimaire an II (19 décembre 1793), "première véritable loi scolaire sur l'enseignement primaire", prescrivait au moins trois années consécutives d'enseignement obligatoire et gratuit pour les garçons et les filles âgés d'au moins six ans. De courte durée, comme le régime jacobin qui l'a promulguée, cette loi fut remplacée par la loi Lakanal du 27 brumaire an III (17 novembre 1794) qui maintint l'obligation et la gratuité de la scolarisation des enfants des deux sexes tout en rendant cette scolarisation plus rigoureuse sur le plan intellectuel et pédagogique. Avec la loi Daunou du 3 brumaire an IV (25 octobre 1795), dont les dispositions pour les écoles primaires restèrent en vigueur jusqu'aux premières années du régime napoléonien, l'enseignement primaire n'était plus gratuit ni obligatoire, mais chaque commune était obligée d'établir une école publique pour les enfants des deux sexes. Voir René Grevet, L'Avènement de l'école contemporaine en France (1789-1835), Villeneuve d'Ascq, Presses universitaires du Septentrion, 2001, p. 59-66 et 69-73, p. 62 pour la citation; et Isser Woloch, The New Regime: Transformations of the French Civic Order, 1789-1820s, New York, Norton, 1994, chapitre 6, en particulier p. 193.

13. Alexis de Jussieu, Histoire de l'instruction primaire en Savoie d'après des archives départementales, communales et paroissiales, Chambéry, A. Perrin, 1875, p. 238-39.

14. Jean Nicolas a publié La Savoie au XVIII' siècle : noblesse et bourgeoisie, Paris, Maloine, 1978, 2 volumes. 
leurs marchandises, surtout des produits de contrebande, et tout le monde échangeait des commérages et les nouvelles. Le cabaret était, pour reprendre l'expression de Jean Nicolas, «un journal oral» ${ }^{15}$.

L'étude de cas nous indique que Nanette connaissait bien le milieu vivant, excitant et éminemment moderne du cabaret, peut-être directement, peutêtre par ce qu'en disaient les autres. Elle indique aussi qu'elle était captivée par le marché. On peut lire cette observation d'une journée de février 1823 passée dans son village : «Souffrances extrêmes jusqu'aux 3 heures après midi. Marchand forain - Nanette examine un quart d'heure sa petite boutique [Puis] grande gaîté jusqu'à $11 \mathrm{~h}$ du soir alors retour de l'état du jour précédent. " C'est une anecdote surprenante et étrangement en avance sur son temps quand on se rappelle l'époque dont elle date (deux décennies après la Révolution) aussi bien que de la couche sociale à laquelle appartient Nanette. La jeune paysanne ressemble presque au stéréotype d'une femme de la classe moyenne de notre époque ayant appris par les journaux féminins la valeur thérapeutique du shopping!

Il y a d'autres exemples du même type. Quand Nanette, tombée dans un état somnambulique entre le sommeil et la veille, va avec Mailland d'Aix à Annecy dans une voiture publique, elle commence une conversation avec les autres voyageurs et étonne tout le monde en déclarant que la canne d'un de ces messieurs est un parapluie. Cette déclaration, apparemment saugrenue, se trouve en fait correcte : la canne convertible en parapluie, combinant ainsi deux accessoires masculins quotidiens, était un nouveau produit de consommation à cette date, et c'est exactement cette "canne à parapluie» que portait ce monsieur ${ }^{16}$. Les autres voyageurs ont précisé que personne dans la diligence n'avait parlé devant Nanette ni de la canne ni de son contenu. Pour sa part, Despine se sert de cet épisode comme preuve de la capacité de Nanette, pendant qu'elle est en crise, de voir sans le secours des yeux. Même Bertrand, ordinairement très méfiant à l'égard des pouvoirs sensoriels extraordinaires que Despine attribue à Nanette dans ses états de somnambulisme, estime cette preuve plus convaincante que celles que son collègue avait l'habitude d'avancer. Autrement dit, les médecins de Nanette jugent plus probable qu'une paysanne comme elle puisse voir à travers le bois utilisé pour fabriquer une canne plutôt qu'elle puisse s'y connaitre en articles de consommation de luxe. Il est cependant évident que Nanette était très attentive à de tels biens et qu'elle en avait vus et observés de près chez les habitués des thermes d'Aix. La "révolution de la consommation" a donc touché Nanette de deux façons : du fait de l'effervescence socio-économique que connaissait toute la

15. Jean Nicolas, La Révolution française dans les Alpes: Dauphiné et Savoie, 1789-1799, Toulouse, Privat, 1989 , p. 31, 34, 39-40.

16. Sur l'invention et l'introduction de la canne à parapluie, voir Jean-Marie Roland de la Platière, «Boursiers», Encyclopédie méthodique : Manufacture, arts et métiers, Paris, Panckoucke, 1785, volume 1, p. 85 et Annie Sagalow, «Le parapluie», dans Les Accessoires du temps : ombrelles, parapluies, Paris, Musée de la mode et du costume, 1990, p. 47-69, en particulier 50-51. 
Savoie (par exemple, le marchand forain qui lui a rendu visite dans son propre village), et du fait qu'elle vivait près de la station thermale d'Aix (où elle a de plus passé un an en internat), qui l'a mise en contact avec des gens riches et cosmopolites momentanément transplantés. Cette proximité lui a aussi fourni le traitement médical savant du docteur Despine (que l'on peut également considérer comme un produit de consommation, mais un produit qui lui a été donné gratuitement et charitablement parce qu'elle était une malade indigente). En l'absence de la station thermale - cette poche incongrue de modernité, comme je l'ai déjà écrit - les charlatans de campagne qui l'ont soignée au début de sa maladie, y compris l'homme que Bertrand baptise ironiquement «l'esculape de village», auraient été ses seuls guérisseurs.

Cette première phase de la révolution de consommation, les historiens comme Daniel Roche l'ont montré, avait un effet généralement euphorisant sur les gens ${ }^{17}$. Phénomène nouveau, l'accès d'une bonne part de la population à des biens de consommation a pu émanciper les individus des anciennes hiérarchies sociales; elle a stimulé leur imagination et leurs désirs, leur faisant imaginer que leur avenir pouvait être différent de leur présent, que la possession de tel ou tel objet pouvait changer leur vie et leur être. Ajoutée à la mémoire récente de la Révolution française, la révolution de la consommation pouvait permettre à quelqu'un comme Nanette d'aspirer à l'autonomie et à l'affirmation de soi comme sujet, aspiration qui pour elle ne s'exprimait que par des symptômes psychopathologiques. La preuve peut-être la plus forte en faveur de cette thèse est la demande de montre de Nanette. Celle-ci n'a en effet pas nommé n'importe quelle montre mais un objet très spécifique : une montre à savonnette, nouveau style très à la mode à l'époque, de forme ronde et légèrement convexe évoquant ainsi un petit morceau de savon moulé pour la toilette. Cette montre avait aussi un double boîtier, l'un de ses deux couvercles de métal protégeant le verre délicat de la face, et un bouton, appelé «le secret», permettant d'ouvrir ce couvercle. À cette date, la montre à savonnette était toujours un produit de luxe, faite en or et richement décorée ${ }^{18}$. On ne sait pas exactement où Nanette a pu apprendre l'existence de ce genre d'objet; mais une image du Journal des dames et des modes de 1824 représente une femme élégante portant une telle montre "en sautoir» - mode que Nanette adoptera après avoir reçu la montre à savonnette de Despine ${ }^{19}$.

Pourquoi Nanette a-t-elle demandé une montre? À cette époque, un tel objet n'était guère un bien ordinaire pour une paysanne; en effet, il fallut attendre les années 1880 pour voir la production en série de montres à bon

17. Voir par exemple Daniel Roche, «Apparences révolutionnaires ou révolution des apparences», dans Modes et révolutions, 1780-1804, Paris, Musée de la mode et du costume, 1989, p. 105-27, p. 111 en particulier.

18. Je dois ces informations sur la montre à savonnette à des communications personnelles de JeanMichel Piguet, conservateur adjoint du Musée international de l'horlogerie à La-Chaux-des-Fonds (Suisse) et d'Estelle Fallet, conservatrice du Musée de l'horlogerie et de l'émaillerie de Genève.

19. Journal des dames et des modes, 15 février 1824, planche 2214. 
marché et leur diffusion dans les campagnes ${ }^{20}$. De plus, Nanette n'avait pas réellement besoin d'une montre. Pour connaître l'heure, la position du soleil et le son du clocher de l'église lui suffisaient amplement. Elle aimait certainement bien l'idée de porter une montre pour "satisfaire à sa vanité", comme le disait Bertrand, et pour apparaitre au-dessus de son rang social. Mais une montre signifiait beaucoup plus pour Nanette : en effet, si on la rapproche d'un autre élément du cas, on comprend très vite que la temporalité en constitue un thème dominant. Cet autre élément, c'est la périodicité menstruelle.

Dans le bref résumé du cas que j’ai présenté, j’ai déjà suggéré cet élément en indiquant, suivant exactement les termes de Despine et de Bertrand, l'âge de Nanette au moment de la première apparition de ses règles. En fait, l'intérêt montré par nos deux médecins-auteurs pour la menstruation de Nanette frise l'obsessionnel. On apprend dans les premiers paragraphes même de l'étude de cas non seulement son âge au début de la menstruation, mais aussi que des interruptions de trois ou quatre mois ont caractérisé son cycle menstruel, que sa menstruation était accompagnée d'une pâleur du visage, que l'attentat à la pudeur du garde champêtre avait lieu «à l'époque de ses règles, qui furent aussitôt supprimées", et ainsi de suite. Tant de petits détails soigneusement rassemblés! En exprimant cette préoccupation, Despine et Bertrand étaient tout à fait représentatifs des médecins français de leur époque, qui avaient tendance à vénérer le flux menstruel comme indicateur clinique privilégié : «le signe et la mesure de la santé de la femme», selon Pierre Roussel, le médecin éclairé dont le livre canonique sur les femmes avait été maintes fois réédité dans la première moitié du XIX ${ }^{\mathrm{e}}$ siècle ${ }^{21}$. Cette préoccupation médicale révèle à son tour une conception essentialiste de la femme considérée uniquement comme procréatrice. Ainsi, après les noces de Nanette, ses médecins toujours exigeants, qui s’inquiétaient jusqu'alors des irrégularités de sa menstruation, ont commencé à s'inquiéter presque immédiatement du phénomène inverse, à savoir du retour ponctuel chaque mois de ses règles : "Nanette est mariée depuis trois mois; elle n'est pas encore enceinte. Les règles ont paru aux époques ordinaires.»

Pour sa part, Nanette n'exprime ouvertement aucun mécontentement à propos des questions répétées et envahissantes de ses médecins sur tous les aspects de sa menstruation mais elle fait la même remarque de façon indirecte, dans le langage de ses symptômes, en demandant à avoir sa propre

20. Catherine Cardinal, La montre des origines au XIX'e siècle, Office du Livre, Fribourg, 1985, p. 13, 47, 52-53, 55-57, 60, 65-67 et Eric Bruton, The History of Clocks and Watches, London, Orbis, 1979, chapitre 9 : «Watches for the People».

21. Voir Pierre Roussel, Système physique et moral de la femme, nouvelle édition, Paris, Fortin, Masson et Cie, 1845, p. 151. Cet ouvrage était publié pour la première fois en 1775, mais sa pertinence continue dans la culture médicale française peut se voir dans une édition de 1803 préparée par l'Idéologue Jean-Louis Alibert et dans cette édition de 1845 du docteur Laurent Cerise, un des fondateurs du premier journal psychiatrique en France. 
montre. Elle demande, en effet, d'être la gardienne de sa propre horloge ou la gérante réfléchie et délibérée de son propre emploi du temps, et non l'être féminin imaginé par ses médecins, gouverné par une temporalité dictée par son corps et incapable d'agir selon sa propre volonté. Si l'on suit la thèse de David Landes dans sa célèbre histoire de la mesure du temps, une montre rendait possible à cette époque ce qu'il appelle «la personnalisation du temps» et "l'élaboration d'un programme personnel »22. Elle fournissait à son porteur un accès permanent et toujours visible à l'heure exacte, situation très nouvelle par rapport à la dépendance traditionnelle des villageois à l'égard du son intermittent du clocher ou de la vue également intermittente de l'horloge sur la grande place. En ce sens, le vif désir de Nanette de posséder une montre est l'image inversée du geste symbolique de Rousseau dans ses Confessions. Après avoir gagné le prix de l'Académie de Dijon pour son Discours sur le progrès des arts et des sciences et avoir vécu, avec moins de plaisir que de dégoût, une période de célébrité, il se résout à réformer sa vie et à se modeler sur la nature. "Je commençai ma réforme par ma parure; je quittai la dorure et les bas blancs, je pris une perruque ronde, je posai l'épée, je vendis ma montre, en me disant avec une joie incroyable : "Grâce au ciel, je n'aurai plus besoin de savoir l'heure qu'il est". ." ${ }^{23}$ Jean-Jacques cherche à embrasser le naturel et à rejeter l'artificiel en se débarrassant de sa montre. Par contraste, Nanette veut une montre pour rompre avec la vie purement naturelle du corps et pour s'insérer dans le flux de temps artificiel et social mesuré par des instruments fabriqués par l'homme.

On peut pousser un peu plus loin cet exercice de phénoménologie historique du temps en citant le beau livre d'Alain Corbin, Les Cloches de la terre. Selon Alain Corbin, le carillonnement qui marquait l'heure et qui faisait diverses autres annonces publiques dans les campagnes au XIX ${ }^{\mathrm{e}}$ siècle était le représentant sonore de la collectivité sociale. Il conférait une identité territoriale aux gens qui vivaient toujours enracinés dans l'espace où ils pouvaient l'entendre ${ }^{24}$. En demandant une montre, Nanette aurait donc exprimé implicitement le souhait de se retirer de cette collectivité, de s'individualiser. Les détails de l'étude du cas confirment très fortement une telle thèse. Tout simplement, Nanette s'identifie à la montre. Quand la montre s'arrête, par exemple, elle succombe à une crise nerveuse; quand on la remonte, sa santé est aussitôt rétablie. Despine note que, pendant un séjour dans son cabinet, Nanette était soucieuse de remonter la montre avec exactitude et de l'examiner plusieurs fois. En plein milieu du cas, il résume ainsi ses observations : «L'influence de la montre sur Nanette et la nécessité des mouvements de ses

22. David S. Landes, Revolution in Time: Clocks and the Making of the Modern World, $2^{\mathrm{e}}$ édition, Cambridge (Mass.), Harvard University Press, 2000, p. 92.

23. Rousseau, Confessions, livre VIII, dans CEuvres complètes, 4 volumes, Paris, Gallimard, "Bibliothèque de la Pléiade», 1959, tome I, p. 363.

24. Alain Corbin, Les Cloches de la terre : paysage sonore et culture sensible dans les campagnes au XIX siècle, Paris, Albin Michel, 1994, chapitre 4. 
rouages pour l'intégrale des fonctions de cette jeune femme furent de nouveau constatés. " Et il faut observer aussi qu'en remplaçant la temporalité naturelle de la menstruation par la temporalité artificielle de la montre, Nanette peut se servir du même vocabulaire : une femme dont la menstruation ne présente aucun problème est bien réglée; une montre qui fait tic-tac et qui affiche l'heure correcte est également bien réglée. Quand la montre cesse de faire tic-tac, Despine emploie le mot «écoulée» pour la caractériser; c'est un mot de la même famille que le verbe qu'il emploie pour décrire la menstruation de Nanette : «Depuis son dernier voyage, Nanette s'est trouvée très bien. Les règles ont coulé avec assez d'abondance.» Ce vocabulaire commun souligne le caractère approprié de l'identification de Nanette à sa montre et la logique de ses efforts pour que le temps artificiel et non pas biologique soit la référence première qui organise et donne un sens à sa vie.

Dans cet article sur l'étude de cas de Nanette Leroux, je me suis concentrée sur deux éléments - les biens de consommation et le temps - parce que je crois qu'ils rendent possible une lecture véritablement historique de ce texte médical. Certes, l'un et l'autre n'ont pas provoqué la maladie de Nanette, mais une fois déclenchée par l'attentat à la pudeur du garde champêtre, cette maladie, comme toute psychopathologie, a utilisé les matériaux fournis par la vie quotidienne de son époque particulière pour élaborer ses symptômes. C'est bien la révolution de la consommation qui a touché la Savoie et surtout la station thermale d'Aix (ainsi que la mémoire de la Révolution française en Savoie) qui ont produit le contexte dans lequel Nanette a pu imaginer une alternative à la vie qu'elle menait. C'était dans l'optique de cette alternative imaginée qu'elle a, d'une part, vécu le geste du garde champêtre et, d'autre part, jugé comme inadéquate la vie traditionnelle d'une femme de la campagne. N'étant pas entièrement consciente de ses sentiments et étant de ce fait incapable de les exprimer dans le langage des mots, elle a «parlé» par le moyen de ses symptômes. En choisissant un produit de consommation très à la mode - la montre à savonnette - comme remède approprié à sa maladie et comme substitut d'elle-même, elle a indiqué son souhait et d'un horizon plus large et d'un rapport moderne à l'expérience du temps.

C'est un truisme souvent dépourvu de contenu des discours universitaires et mondains sur le consumérisme de nos jours que d'affirmer que les gens construisent leurs identités autour des biens de consommation qui leur sont accessibles sur le marché. Lu avec un regard historique, le cas de Nanette Leroux nous fournit un exemple concret, nuancé et soigneusement développé de cette même thèse. Il atteste que dans les années 1820 et dans la région périphérique et agricole qu'était alors la Savoie, en un temps et une région au seuil de la modernité, la thèse pouvait avoir déjà une certaine validité et que les identités ainsi imaginées pouvaient avoir un effet déstabilisateur. 